\title{
A saúde bucal das comunidades indígenas brasileiras: uma revisão integrativa
}

\author{
Oral health of brazilian indigenous communities: an integrative review \\ Salud oral de las comunidades indígenas brasileñas: una revisión integradora
}

Recebido: 18/12/2020 | Revisado: 19/12/2020 | Aceito: 21/12/2020 | Publicado: 01/01/2021

\author{
Diogo Gomes Brandão \\ ORCID: https://orcid.org/0000-0001-6167-0399 \\ Universidade Federal de Alagoas, Brasil \\ E-mail: diogo.brandao2009@hotmail.com \\ Jéssica Stherphanny Medeiros de Oliveira Moraes \\ ORCID: https://orcid.org/0000-0002-0305-4229 \\ Universidade Federal de Alagoas, Brasil \\ E-mail: contato.jessicamedeiros@outlook.com \\ Dayse Andrade Romão \\ ORCID: https://orcid.org/0000-0002-7884-1657 \\ Universidade Federal de Alagoas, Brasil \\ E-mail: dayseromao@gmail.com
}

\begin{abstract}
Resumo
Segundo o Instituto Brasileiro de Geografia e Estatística (IBGE), o Brasil possui mais de 240 comunidades indígenas. As características etnográficas dessas comunidades corroboraram para uma grande variedade de determinantes do processo saúde-doença, onde a efetividade das ações de atenção à saúde básica é uma missão desafiadora. Este trabalho trata-se de uma revisão integrativa, que objetivou analisar estudos sobre as condições de saúde bucal dos povos indígenas brasileiros, seus objetivos e resultados. Os artigos compilados neste estudo foram selecionados por meio das bases de dados: PubMed, Lilacs e Scielo. O levantamento limitou-se aos artigos publicados nos idiomas inglês e português, entre os anos de 2000 a 2020, utilizando os descritores padronizados: Saúde Bucal; Saúde Indígena; Índios Sul-Americanos e seus correspondentes em inglês, em todas as combinações foi utilizado o operador booleano And. Para que pudessem serem incluídos na análise, utilizou-se os seguintes critérios: estar em formato de artigo, possuírem título e resumo que se assemelhem aos objetivos do trabalho e estar publicado na íntegra. Observouse que devido as peculiaridades sócio-demográficas, cercearam muitos destes aos benefícios de programas assistenciais odontológicos de rotina, tendo como consequência um controle insipiente da cárie e desenvolvimento de várias doenças bucais. Contudo, ficou evidente que a condição de saúde bucal dos povos indígenas brasileiros apresenta-se precária e que essa condição de degradação só poderá ser dirimida por meio de estudos epidemiológicos mais abrangentes, além da execução de ações de prevenção e promoção à saúde bucal.
\end{abstract}

Palavras-chave: Saúde bucal; Saúde indígena; Índios sul-americanos.

\begin{abstract}
According to the Brazilian Institute of Geography and Statistics (IBGE), Brazil has more than 240 indigenous communities. The ethnographic characteristics of these communities corroborated for a wide variety of determinants of the health-disease process, where the effectiveness of basic health care actions is a challenging mission. This work is an integrative review, which aimed to analyze studies on the oral health conditions of Brazilian indigenous peoples, their objectives and results. The articles compiled in this study were selected through the databases: PubMed, Lilacs and Scielo. The survey was limited to articles published in English and Portuguese, between 2000 and 2020, using the standardized descriptors: Oral Health; Indigenous Health; South American Indians and their English counterparts, the Boolean operator And was used in all combinations. In order to be included in the analysis, the following criteria were used: be in article format, have title and abstract that are similar to the objectives of the work and be published in full. It was observed that due to socio-demographic peculiarities, many of these have been restricted to the benefits of routine dental care programs, resulting in an insipient control of caries and the development of various oral diseases. However, it was evident that the oral health condition of Brazilian indigenous peoples is precarious and that this condition of degradation can only be resolved through more comprehensive epidemiological studies, in addition to the implementation of preventive and oral health promotion actions.
\end{abstract}

Keywords: Oral health; Indigenous health; South american indians.

\section{Resumen}

Según el Instituto Brasileño de Geografía y Estadística (IBGE), Brasil tiene más de 240 comunidades indígenas. Las características etnográficas de estas comunidades se corroboran para una amplia variedad de determinantes del proceso salud-enfermedad, donde la efectividad de las acciones básicas de atención en salud es una misión desafiante. Este trabajo es una revisión integradora, que tuvo como objetivo analizar los estudios sobre las condiciones de salud 
bucal de los pueblos indígenas brasileños, sus objetivos y resultados. Los artículos recopilados en este estudio fueron seleccionados a través de las bases de datos: PubMed, Lilacs y Scielo. La encuesta se limitó a artículos publicados en inglés y portugués, entre 2000 y 2020, utilizando los descriptores estandarizados: salud bucal; Salud Indígena; Indios sudamericanos y sus contrapartes inglesas, el operador booleano And se usó en todas las combinaciones. Para ser incluido en el análisis se utilizaron los siguientes criterios: estar en formato de artículo, tener título y resumen similar a los objetivos del trabajo y estar publicado en su totalidad. Se observó que debido a peculiaridades sociodemográficas, muchas de estas se han restringido a los beneficios de los programas de atención odontológica rutinaria, resultando en un control insipiente de la caries y el desarrollo de diversas enfermedades bucales. Sin embargo, se evidenció que la condición de salud bucal de los pueblos indígenas brasileños es precaria y que esta condición de degradación solo puede resolverse mediante estudios epidemiológicos más integrales, además de la implementación de acciones preventivas y de promoción de la salud bucal.

Palabras clave: Salud bucal; Salud indígena; Indios sudamericanos.

\section{Introdução}

A busca pela integralidade do cuidado a pessoa humana, reconhecendo as diferenças nas condições sociais, de acordo com as reais necessidades dos indivíduos, é o principal alicerce da Constituição Federal de 1988. Neste contexto, sabemos que o Brasil, além de proporções continentais possui uma população extremamente diversificada entre suas regiões (Neves, Giordani e Hugo, 2019; Galea e Vaughan, 2019). Dentre elas, a população indígena possui inúmeras particularidades que estão ligadas a fatores culturais, ambientais, políticas, biológicas e econômicas (Souza et al., 2019).

Segundo o Censo Demográfico do Instituto Brasileiro de Geografia e Estatística (IBGE) de 2010, no Brasil existem mais de 305 povos indígenas que somam aproximadamente 896.917 pessoas. Destes, em torno de 324.834 vivem em cidades e 572.083 em áreas rurais, o que corresponde aproximadamente a 0,47\% da população total do país (Wenczenovicz, 2018). Para além das estatísticas demográficas, a população indígena em todos seus aspectos e particularidades culturais, contribuíram significativamente para a construção iconográfica do povo brasileiro (Nicácio et al., 2019; Silva, 2019).

Entretanto, quando busca-se setorializar a efetividade das ferramentas de atenção básica à saúde para as comunidades indígenas brasileiras, sobretudo os cuidados com a saúde bucal, verificamos uma lacuna significativa nesses atendimentos (Baldisserotto, Ferreira e Warmling, 2019). É possível ainda, identificar que existem vários entraves que dificultam a consonância desses cuidados, são eles: fatores demográficos, processos históricos de interações com as sociedades nãoindígenas, difícil acesso aos serviços de saúde, as barreiras linguísticas e as tecnológicas utilizadas pelos serviços de saúde (Garnelo, Brandão e Levino, 2005).

Compreende-se que o cuidado integral à saúde bucal é algo inerente e extremamente necessário ao indivíduo (França et al., 2020; Ferreira et al., 2020). Para a odontologia moderna, a doença cárie e as periodontopatias são as afecções mais prevalentes e alvo primário das estratégias de saúde básica, diante disso, a população indígena brasileira tem se tornado um grupo alvo de preocupação, devido aos dados alarmantes de má condição bucal, alta prevalência de perdas dentárias, além das limitações inatas aos serviços de prevenção e tratamento odontológico (Junior et al., 2020; Netto e Chicon, 2020).

São escassos os registros sobre a atenção em saúde bucal indígena, apenas com a implantação do programa federal Brasil Sorridente, no final do ano 2003 possibilitou um maior acesso ao atendimento odontológico, estruturando e qualificando os serviços de saúde bucal e garantindo assistência odontológica integral a esta população. Antes desse programa, as ações em favor as comunidades indígenas eram localizadas e sutis, caracterizadas pela imensa fragmentação e descontinuidade do cuidado, além de serem predominantemente curativas, sem a possibilidade de tratamentos mais complexos e multiprofissionais (Tavares et al., 2020).

Contudo, é imprescindível compreender que um paciente indígena traz consigo sua própria significação do mundo, da vida, da morte e das causas espirituais sobre as enfermidades (Kabad, Pontes e Monteiro, 2020). Entender as interpretações 
desse povo, corroborará para uma percepção particular do contexto saúde-doença que promoverá uma interessante reflexão acerca da formação do Cirurgião-Dentista, dentro do exercício de sua prática profissional (Lima et al., 2020).

Em consonância ao exposto, justifica-se este estudo para análise das evidências científicas sobre a compreensão dos avanços e entraves da prática odontológica nas áreas indígenas, pois este grupo tem se tornado alvo de preocupação devido suas condições precárias de saúde bucal, além de haver uma escassez de estudos que relatem a epidemiologia dessas populações.

\section{Metodologia}

O presente trabalho trata-se de uma revisão integrativa, que é uma modalidade de estudo que proporciona uma síntese de conhecimento e a incorporação da aplicabilidade de resultados de estudos significativos na prática de uma determinada área, a partir da formulação de uma pergunta, identificação, seleção e avaliação crítica dos resultados (Souza, Silva e Carvalho, 2010).

Foi realizada uma pesquisa bibliográfica sobre o tema central que envolveu a seguinte pergunta norteadora: Qual $o$ perfil epidemiológico da saúde bucal dos povos indígenas brasileiros?

A partir da utilização das bases de dados PUBMED, LILACS, e SCIELO foi realizada a seleção de trabalhos entre abril de 2020 a agosto de 2020, utilizando os descritores padronizados pelo Descritores em Ciências da Saúde (Decs): Saúde Bucal; Saúde Indígena; Índios Sul-Americanos, e seus correspondentes em inglês. Em todas as combinações foi utilizado o operador booleano And. O levantamento limitou-se aos artigos publicados em língua portuguesa e inglesa, entre os anos de 2000 e 2020.

Os estudos foram compilados inicialmente pelos títulos, refinados pelos resumos. Após leitura dos resumos, os artigos que indicavam corresponder ao objetivo desta revisão, foram lidos integralmente, e uma vez que preencheram os critérios de inclusão, participaram deste estudo.

Desta forma, para que os artigos encontrados nas bases de dados pudessem serem incluídos na análise, utilizaram-se os seguintes critérios: estar em formato de artigo, possuírem título e resumo que se assemelhem aos objetivos do trabalho e estar publicado na íntegra. Excluíram-se todos os trabalhos que não corresponderam a estes critérios, conforme ilustrado na Figura 1. 
Figura 1: Etapas da coleta de dados de estudo.

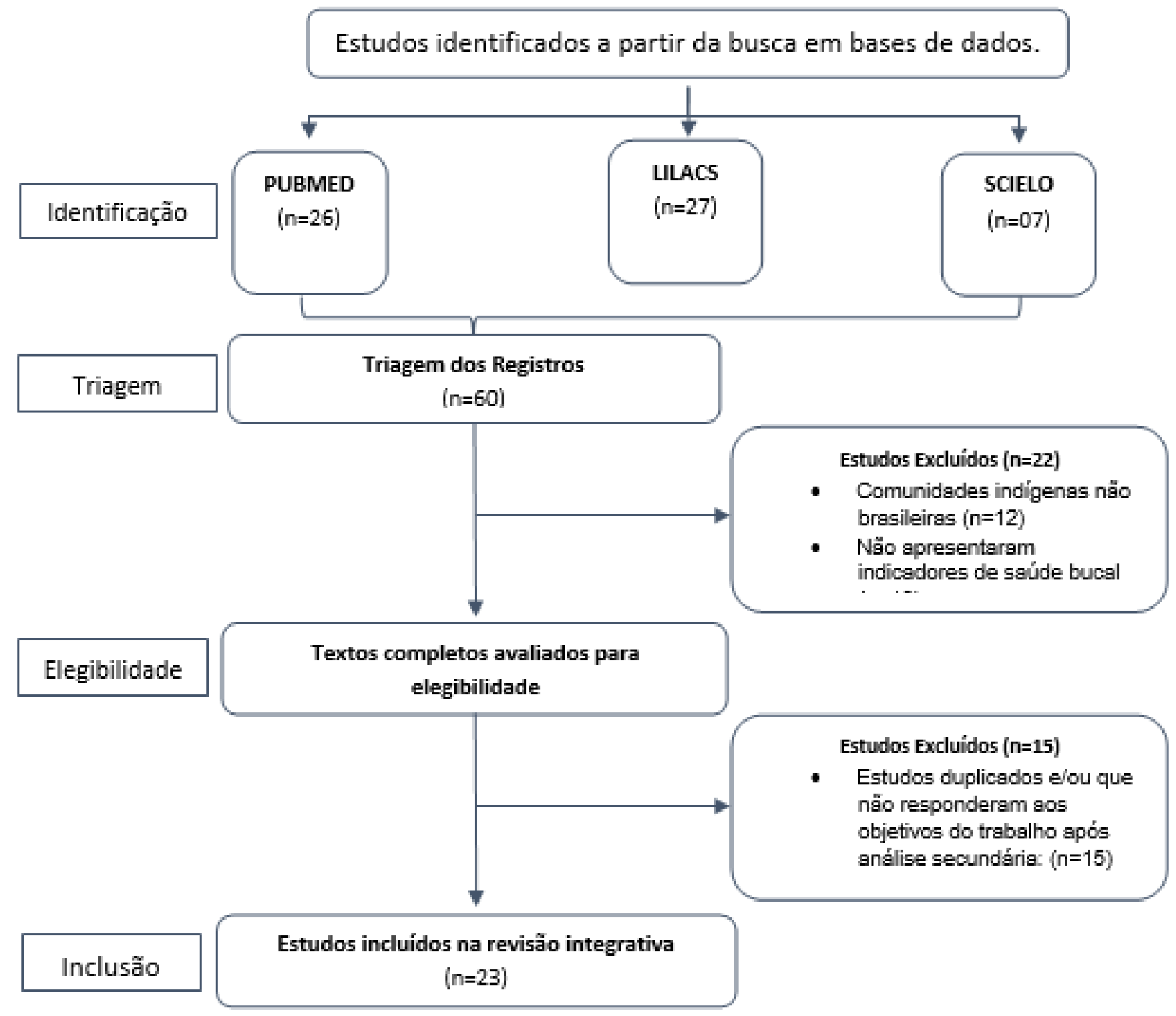

Fonte: Autores (2020).

\section{Resultados}

Utilizando as palavras Saúde Bucal, Saúde Indígena e Índios Sul-Americanos, foram encontrados um total de 60 artigos nas bases de dados PubMed, Lilacs e Scielo, nos idiomas escolhidos. Sendo 13 artigos destes excluídos na leitura de título, dos 47 trabalhos que restaram, 9 foram excluídos na leitura de resumos. Ao final, 38 artigos estavam disponíveis para leitura na íntegra, onde destes, 15 foram excluídos por repetição. Ao final, restaram 23 publicações que estavam de acordo com os critérios de inclusão do presente estudo.

Os estudos analisados no Quadro 1, mostraram que o interesse em mapear o perfil da saúde bucal das comunidades indígenas brasileiras ainda é algo muito insipiente. Ao visualizar os trabalhos realizados nos últimos 20 anos, deve-se considerar que o percurso da saúde bucal indígena brasileira é fortemente marcada por dissidências, o que reflete a necessidade de conduzir estudos epidemiológicos em saúde bucal mais robustos e abrangentes com essas comunidades. 
Quadro 1: Síntese de publicações incluídas na revisão integrativa, entre os anos 2000 e 2020 segundo as bases de dados PubMed, Lilacs e Scielo.

\begin{tabular}{|c|c|c|c|c|}
\hline TÍTULO E AUTORES & ANO & DELINEAMENTO & OBJETIVOS & DESFECHO \\
\hline $\begin{array}{l}\text { Some aspects of the } \\
\text { conditions of Oral Health } \\
\text { of the population } \\
\text { indigenous Guarani Mbyá } \\
\text { in the Municipal district } \\
\text { of Säo Paulo } \\
\text { (Fratucci) }\end{array}$ & 2000 & $\begin{array}{c}\text { Estudo } \\
\text { Qualitativo }\end{array}$ & $\begin{array}{l}\text { Realizar diagnóstico de saúde } \\
\text { bucal da comunidade indígena } \\
\text { Guarani, Mbyá da Aldeia } \\
\text { Morro da Saudade }\end{array}$ & $\begin{array}{l}\text { Considerando os indivíduos examinados, } \\
\text { pode-se observar que o perfil } \\
\text { epidemiológico das doenças bucais tem } \\
\text { um comportamento diferenciado da } \\
\text { sociedade não indígena, embora não } \\
\text { exista acesso a programas preventivos e } \\
\text { assistenciais. }\end{array}$ \\
\hline $\begin{array}{l}\text { Oral health among the } \\
\text { Xavánte Indians in } \\
\text { Pimentel Barbosa, Mato } \\
\text { Grosso, Brazil } \\
\text { (Arantes, Santos e } \\
\text { Coimbra) }\end{array}$ & 2001 & Estudo Descritivo & $\begin{array}{l}\text { Realizar uma comparação } \\
\text { com o estudo epidemiológico } \\
\text { em saúde bucal que foi } \\
\text { execultado no ano de 1997, } \\
\text { afim de compreender o } \\
\text { comportamento desses } \\
\text { indicadores na comunidade } \\
\text { indígena Xavánte de } \\
\text { Etéñitépa. }\end{array}$ & $\begin{array}{l}\text { Apresentaram que as mudanças na dieta } \\
\text { devido a alterações ambientais e } \\
\text { socioeconômicas, além da falta de } \\
\text { programas preventivos, estão entre as } \\
\text { causas dessa deterioração da saúde bucal } \\
\text { entre os Xavánte. }\end{array}$ \\
\hline $\begin{array}{l}\text { Dental caries experience } \\
\text { in Indians of the Upper } \\
\text { Xingu, Brazil } \\
\text { (Rigonatto, Antunes e } \\
\text { Frazão) }\end{array}$ & 2001 & $\begin{array}{c}\text { Estudo Transversal } \\
\text { Descritivo }\end{array}$ & $\begin{array}{l}\text { Descrever a experiência de } \\
\text { cárie nas comunidades } \\
\text { indígenas do Xingu. }\end{array}$ & $\begin{array}{l}\text { O estudo indicou baixa incorporação de } \\
\text { serviços odontológicos, além de questões } \\
\text { como a irregularidade dos serviços } \\
\text { programados para essas comunidades, as } \\
\text { mudanças nos padrões alimentares e } \\
\text { culturais, reforça a necessidade premente } \\
\text { de iniciativas de promoção da saúde } \\
\text { voltadas para esses grupos. }\end{array}$ \\
\hline $\begin{array}{l}\text { A view about the Baniwa } \\
\text { Indians: oral health and } \\
\text { dentistry attention in the } \\
\text { region from Alto Rio } \\
\text { Negro, Brazilian } \\
\text { Amazonia, } 2000 \text { to } 2004 \\
\text { (Carneiro) }\end{array}$ & 2005 & $\begin{array}{c}\text { Estudo } \\
\text { Qualitativo }\end{array}$ & $\begin{array}{l}\text { Conhecer aspectos } \\
\text { relacionados à atenção } \\
\text { odontológica oferecida a } \\
\text { comunidade Baniwa dopólo- } \\
\text { base de Tunuí-Cachoeira, São } \\
\text { Gabriel da Cachoeira, } \\
\text { Amazonas. }\end{array}$ & $\begin{array}{l}\text { Do total de pessoas examinadas, } 73,6 \% \\
\text { apresentaram alguma necessidade de } \\
\text { tratamento cirúrgico restaurador. Os } \\
\text { indivíduos entre 15-19 anos apresentam } \\
\text { as mais elevadas frequências de } \\
\text { restaurações. Enfatiza-se a necessidade } \\
\text { de ampliação da atenção à saúde bucal, } \\
\text { considerando-se a complexidade } \\
\text { sociocultural destes povos. }\end{array}$ \\
\hline $\begin{array}{l}\text { Evaluation of molar and } \\
\text { incisor bite force in } \\
\text { indigenous compared } \\
\text { with white population in } \\
\text { Brazil } \\
\text { (Regalo et al.) }\end{array}$ & 2008 & $\begin{array}{c}\text { Estudo } \\
\text { Qualitativo }\end{array}$ & $\begin{array}{l}\text { Determinar a força máxima de } \\
\text { mordida nas regiões molares e } \\
\text { incisivas em jovens indígenas } \\
\text { brasileiros, que fazem dieta } \\
\text { natural desde o nascimento, e } \\
\text { comparar a amostra com } \\
\text { indivíduos brasileiros brancos }\end{array}$ & $\begin{array}{l}\text { Comparando indígenas com indivíduos } \\
\text { brancos, a força máxima de mordida } \\
\text { mostrou uma tendência de ser maior no } \\
\text { grupo indígena. Observou-se que a região } \\
\text { dos incisivos apresentava significância } \\
\text { estatística, embora nenhuma significância } \\
\text { foi analisada em região molar. Além } \\
\text { disso, os homens indígenas apresentaram } \\
\text { os maiores valores de força de mordida. }\end{array}$ \\
\hline $\begin{array}{l}\text { Caries, gender and socio- } \\
\text { economic change in the }\end{array}$ & & & $\begin{array}{l}\text { Investigou-se a incidência de } \\
\text { cárie em uma comunidade }\end{array}$ & $\begin{array}{l}\text { O aumento da idade e diferenças entre } \\
\text { sexos são possíveis fatores associados ao }\end{array}$ \\
\hline
\end{tabular}




\begin{tabular}{|c|c|c|c|c|}
\hline $\begin{array}{l}\text { Xavante Indians from } \\
\text { Central Brazil } \\
\text { (Arantes et al.) }\end{array}$ & 2009 & $\begin{array}{c}\text { Estudo Longitudinal } \\
\text { Descritivo }\end{array}$ & $\begin{array}{l}\text { indígena do Brasil Central, } \\
\text { com foco nas diferenças de } \\
\text { gênero. }\end{array}$ & $\begin{array}{l}\text { aumento da cárie dentária e doenças } \\
\text { periodontais na comunidade dos } \\
\text { Xavantes. }\end{array}$ \\
\hline $\begin{array}{l}\text { Oral health in transition: } \\
\text { the case of Indigenous } \\
\text { peoples from Brazil } \\
\text { (Arantes, Santos e } \\
\text { Frazão) }\end{array}$ & 2010 & $\begin{array}{c}\text { Estudo Transversal } \\
\text { Descritivo }\end{array}$ & $\begin{array}{l}\text { Caracterizar informações } \\
\text { epidemiológicas sobre a } \\
\text { distribuição de cárie dentária } \\
\text { entre os povos indígenas no } \\
\text { Brasil. }\end{array}$ & $\begin{array}{l}\text { Foi constatado uma redução nos índices } \\
\text { de cárie dessa comunidade, porém o caso } \\
\text { Xavante é um excelente exemplo da } \\
\text { transição na saúde bucal que está } \\
\text { ocorrendo entre os indígenas } \\
\text { povos das Américas, e destaca a } \\
\text { importância da promoção da saúde bucal } \\
\text { por meio medidas preventivas. }\end{array}$ \\
\hline $\begin{array}{l}\text { Dental caries and } \\
\text { treatment needs among } \\
\text { indigenous people of the } \\
\text { Potiguara Indian } \\
\text { reservation in Brazil } \\
\text { (Sampaio et al.) }\end{array}$ & 2010 & $\begin{array}{c}\text { Estudo Transversal } \\
\text { Descritivo }\end{array}$ & $\begin{array}{l}\text { Avaliar a prevalência de cárie } \\
\text { e as necessidades de } \\
\text { tratamento de residentes } \\
\text { indígenas brasileiros em uma } \\
\text { reserva indígena. }\end{array}$ & $\begin{array}{l}\text { Em todos os grupos da comunidade } \\
\text { Potiguara foi observado uma alta } \\
\text { prevalência de cárie e muitos fatores que } \\
\text { precisam de tratamento. }\end{array}$ \\
\hline $\begin{array}{l}\text { Indigenous peoples: } \\
\text { reflection about the } \\
\text { influence of urban } \\
\text { civilization on nutritional } \\
\text { status and oral health } \\
\text { (Moura et al.) }\end{array}$ & 2010 & Revisão Integrativa & $\begin{array}{l}\text { Identificar a influência da } \\
\text { transição alimentar no estado } \\
\text { nutricional e na condição } \\
\text { bucal da população } \\
\text { indígena brasileira. }\end{array}$ & $\begin{array}{l}\text { Os estudos revelaram a prevalência de } \\
\text { desnutrição infantil, sobrepeso e } \\
\text { obesidade em adultos, índice de cárie } \\
\text { elevado e doença periodontal nas } \\
\text { comunidades indígenas que estão } \\
\text { passando por mudanças de hábitos } \\
\text { culturais, como a transição alimentar. }\end{array}$ \\
\hline $\begin{array}{l}\text { Evaluation of oral health } \\
\text { in a community of native } \\
\text { Brazilians of the Umutina } \\
\text { Reservation, Mato Grosso } \\
\text { State } \\
\text { (Vieira et al.) }\end{array}$ & 2011 & Estudo Qualitativo & $\begin{array}{l}\text { Avaliar a distribuição das } \\
\text { lesões e variações anatômicas } \\
\text { em oito grupos étnicos da } \\
\text { Reserva Indígena Umutina, } \\
\text { através de exames físicos e } \\
\text { orais, além de avaliação } \\
\text { citológica e avaliação de dieta } \\
\text { e hábitos. }\end{array}$ & $\begin{array}{l}\text { Foram examinados índios, de ambos os } \\
\text { sexos, com idades variando de } 1 \text { a } 96 \\
\text { anos, sendo identificadas } 132 \text { variações } \\
\text { da normalidade. Destacando-se uma alta } \\
\text { ocorrência de anquiloglossia, que esteve } \\
\text { presente em } 108 \text { casos ( } 37,1 \%) \text {. } \\
\text { Nenhuma lesão maligna foi identificada } \\
\text { na população. }\end{array}$ \\
\hline $\begin{array}{l}\text { Dental fluorosis and } \\
\text { periodontal conditional } \\
\text { Aracruz, ES, county } \\
\text { village indigenous } \\
\text { children } \\
\text { (Alencar et al.) }\end{array}$ & 2011 & $\begin{array}{c}\text { Estudo } \\
\text { Quantitativo }\end{array}$ & $\begin{array}{l}\text { Avaliar a prevalência de } \\
\text { alterações gengivais, condição } \\
\text { periodontal e fluorose } \\
\text { dentária em crianças indígena } \\
\text { s da etnia Tupiniquim e } \\
\text { Guarani do município de } \\
\text { Aracruz (ES). }\end{array}$ & $\begin{array}{l}\text { Foi observada fluorose dentária nos graus } \\
\text { mais leves da doença de }(26,8 \%) \text {. Em } \\
(64,9 \%) \text { das crianças de } 5 \text { anos não foi } \\
\text { observado sangramento gengival, } \\
\text { entretanto a presença de sangramento foi } \\
\text { verificada em }(68,3 \%) \text { da amostra aos } 12 \\
\text { anos. Os achados deste estudo } \\
\text { permitiram inferir que é premente a } \\
\text { necessidade de implementação de } \\
\text { medidas em saúde bucal para esta } \\
\text { população. }\end{array}$ \\
\hline $\begin{array}{lr}\text { Dentistry } & \text { students' } \\
\text { perceptions } & \text { about an } \\
\text { extramural } & \text { experience } \\
\text { with a } & \text { Brazilian } \\
\text { indigenous } & \end{array}$ & 2012 & Estudo Qualitativo & $\begin{array}{l}\text { Avaliar as percepções dos } \\
\text { estudantes de odontologia } \\
\text { sobre uma atividade } \\
\text { extramuros projetada para } \\
\text { fornecer } \\
\text { atendimento }\end{array}$ & $\begin{array}{l}\text { A participação em um projeto extramuro } \\
\text { melhora a compreensão dos estudantes de } \\
\text { odontologia sobre cuidados primários } \\
\text { para comunidade indígena. }\end{array}$ \\
\hline
\end{tabular}




\begin{tabular}{|c|c|c|c|c|}
\hline $\begin{array}{l}\text { 2012community } \\
\text { (Bulgarelli et al.) }\end{array}$ & & & $\begin{array}{l}\text { odontológico a uma } \\
\text { comunidade indígena. }\end{array}$ & \\
\hline $\begin{array}{l}\text { Oral health care in } \\
\text { indigenous communities: } \\
\text { evolution and challenges } \\
\text { - a literature review } \\
\text { (Bertanha et al.) }\end{array}$ & 2012 & Revisão Integrativa & $\begin{array}{l}\text { Apontar os aspectos relativos } \\
\text { à atenção à saúde bucal } \\
\text { nas comunidades indígenas, } \\
\text { destacando-se a evolução e os } \\
\text { desafios. }\end{array}$ & $\begin{array}{l}\text { Ficou evidente a necessidade do } \\
\text { profissional adquirir competências } \\
\text { culturais, transcendendo os limites de um } \\
\text { modelo assistencialista com foco no } \\
\text { individuo, privilegiando a prevenção com } \\
\text { o olhar voltado à coletividade dos povos } \\
\text { indígenas. }\end{array}$ \\
\hline $\begin{array}{l}\text { The oral health of the } \\
\text { indigenous people } \\
\text { Xukuru do Ororubá in } \\
\text { age group 10-14 years. } \\
\text { (Maurício) }\end{array}$ & 2012 & $\begin{array}{l}\text { Estudo de Corte } \\
\text { Transversal }\end{array}$ & $\begin{array}{l}\text { Analisar a ausência de cárie e } \\
\text { fatores associados do povo } \\
\text { indígena Xukuru do Ororubá } \\
\text { na faixa etária de } 10 \text { a } 14 \text { anos }\end{array}$ & $\begin{array}{l}\text { O perfil epidemiológico bucal, apresenta } \\
\text { alta necessidade de tratamento } \\
\text { odontológico, além do estudo ter } \\
\text { revelado uma alta parcela desta } \\
\text { população que nunca foi ao dentista. }\end{array}$ \\
\hline $\begin{array}{l}\text { Social and environmental } \\
\text { inequities in dental caries } \\
\text { among indigenous } \\
\text { population in Brazil: } \\
\text { evidence from } 2000 \text { to } \\
2007 \\
\text { (Filho, Santos e Vettore) }\end{array}$ & 2013 & Revisão Integrativa & \begin{tabular}{lr} 
O buscou & investigar a \\
associação & \multicolumn{2}{c}{ entre } \\
desigualdades & \multicolumn{2}{c}{ sociais e } \\
ambientais e cárie dentária \\
entre povos indígenas no \\
Brasil.
\end{tabular} & $\begin{array}{l}\text { Ficou evidente que os aspectos } \\
\text { relacionados à localização e e } \\
\text { infraestrutura existente nas comunidades } \\
\text { indígenas, que se vinculam à } \\
\text { disponibilidade de serviços de saúde } \\
\text { bucal, estão associados à ocorrência de } \\
\text { cárie dentária. }\end{array}$ \\
\hline $\begin{array}{l}\text { Oral health status of the } \\
\text { ethnic group Xukuru } \\
\text { from Ororubá: multilevel } \\
\text { analysis } \\
\text { (Maurício e Moreira) }\end{array}$ & 2014 & $\begin{array}{c}\text { Estudo } \\
\text { Qualitativo }\end{array}$ & $\begin{array}{l}\text { Analisar o estado de saúde } \\
\text { bucal dos indígenas Xukuru } \\
\text { de Ororubá de } 10 \text { a } 14 \text { anos, } \\
\text { em Pernambuco, Brasil. }\end{array}$ & $\begin{array}{l}\text { A existência de mais que o dobro de } \\
\text { dentes cariados por indígena, quando } \\
\text { comparados com os indicadores da } \\
\text { população não indígena de Recife é um } \\
\text { provável reflexo da dificuldade de acesso } \\
\text { aos serviços de saúde. }\end{array}$ \\
\hline $\begin{array}{l}\text { Dental caries in mother- } \\
\text { child pairs from Xingu } \\
\text { (Hirooka et al.) }\end{array}$ & 2014 & $\begin{array}{l}\text { Estudo } \\
\text { Quantitativo }\end{array}$ & $\begin{array}{l}\text { Descrever casos de cárie } \\
\text { dentária em pares mãe-filho } \\
\text { indígenas do Parque Xingu. }\end{array}$ & $\begin{array}{l}\text { A análise do índice CPOD mostrou um } \\
\text { valor médio de } 14,3 \text { para as mães } \\
\text { examinadas, entre elas com idade entre } \\
35 \text { e } 44 \text { anos, enquanto às crianças, o } \\
\text { índice ceo-d foi de } 4,7 \text { em média, e } \\
\text { apenas } 13,4 \% \text { eram livres de cárie. } \\
\text { Nenhuma correlação significativa foi } \\
\text { encontrada entre a cárie materna e } \\
\text { infantil. }\end{array}$ \\
\hline $\begin{array}{l}\text { Dental occlusion in } \\
\text { indigenous population } \\
\text { (Campos et al.) }\end{array}$ & 2017 & Revisão Integrativa & $\begin{array}{l}\text { Avaliar os aspectos } \\
\text { sobre oclusão } \\
\text { dentária de povos } \\
\text { indígenas no Brasil. }\end{array}$ & $\begin{array}{l}\text { Nos estudos encontrados, observa se a } \\
\text { diversidade de padrões relacionados à } \\
\text { oclusão dentária. Sendo assim, é } \\
\text { necessário a realização de mais trabalhos } \\
\text { para conhecer o perfil epidemiológico } \\
\text { das populações indígenas no Brasil no } \\
\text { que se refere às oclusopatias, buscando } \\
\text { uma padronização metodológica. }\end{array}$ \\
\hline $\begin{array}{l}\text { Subjective oral symptoms } \\
\text { associated with self-rated } \\
\text { oral health among } \\
\text { Indigenous groups in } \\
\text { Central-West Brazil }\end{array}$ & 2018 & Estudo Transversal & $\begin{array}{l}\text { Determinar se os } r \text { sintomas } \\
\text { orais subjetivos } r \text { foram } \\
\text { associados à autoavaliação da } \\
\text { saúde bucal em quatro } \\
\text { diferentes povos indígenas do }\end{array}$ & $\begin{array}{l}\text { A autoavaliação de saúde bucal, } \\
\text { classificada como ruim, foi mais comum } \\
\text { nas faixas etárias mais avançadas dos } \\
\text { povos Guarani. Confirmando assim que } \\
\text { os sintomas orais como dor de dente e } \\
\text { dificuldade para falar devido a problemas }\end{array}$ \\
\hline
\end{tabular}




\begin{tabular}{|c|c|c|c|c|}
\hline (Arantes e Frazão) & & & Centro-Oeste do Brasil. & $\begin{array}{l}\text { bucais, desempenham um papel } \\
\text { importante na autoavaliação da saúde } \\
\text { bucal. }\end{array}$ \\
\hline $\begin{array}{l}\text { Oral health care in the } \\
\text { Xingu Indigenous Park, } \\
\text { Brazil, from } 2004 \text { to } \\
\text { 2013: an analysis based } \\
\text { on evaluation indicators } \\
\text { (Lemos et al.) }\end{array}$ & 2018 & $\begin{array}{l}\text { Estudo } \\
\text { Quantitativo }\end{array}$ & $\begin{array}{l}\text { Analisar no Parque Indígena } \\
\text { do Xingu de } 2004 \text { a } 2013 \text { os } \\
\text { indicadores de saúde bucal } \\
\text { desta comunidade. }\end{array}$ & $\begin{array}{l}\text { A prevalência de cárie foi considerada } \\
\text { alta em crianças, com redução no grupo } \\
\text { dos adolescentes (15-19 anos). O } \\
\text { panorama dos cuidados odontológicos } \\
\text { dessa comunidade decresceu. }\end{array}$ \\
\hline $\begin{array}{l}\text { Epidemiological analysis } \\
\text { and need for endodontic } \\
\text { treatment among the } \\
\text { indigenous Sateré-Mawé } \\
\text { and Tikuna } \\
\text { (Caires et al.) }\end{array}$ & 2018 & $\begin{array}{l}\text { Estudo } \\
\text { Quantitativo }\end{array}$ & 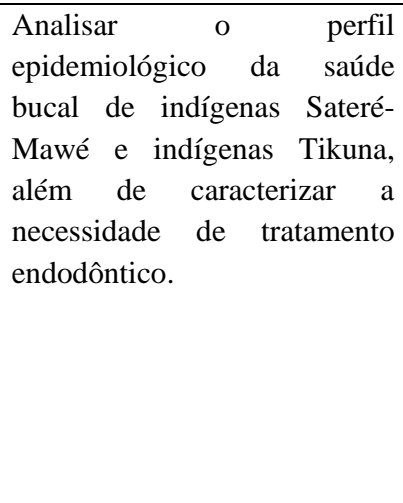 & $\begin{array}{l}\text { A comunidade Sateré-Mawé apresentou } \\
\text { uma maior prevalência de necessidade de } \\
\text { tratamento endodôntico em comparação } \\
\text { com os povos Tikuna. A associação de } \\
\text { comorbidades e a necessidade de } \\
\text { tratamento endodôntico foram } \\
\text { demonstrados apenas nos Tikuna, com } \\
\text { uma correlação desta necessidade com a } \\
\text { presença de diabetes mellitus (DM) em } \\
\text { um caso. Corroborando para importância } \\
\text { da ampliação do acesso as campanhas de } \\
\text { saúde. }\end{array}$ \\
\hline $\begin{array}{l}\text { Análise documental dos } \\
\text { serviços de saúde bucal } \\
\text { ofertados à população } \\
\text { indígena no Brasil } \\
\text { (Rodrigues et al.) }\end{array}$ & 2018 & $\begin{array}{c}\text { Estudo Descritivo } \\
\text { Exploratório }\end{array}$ & $\begin{array}{l}\text { Analisar a política de } \\
\text { saúde bucal inserida no } \\
\text { subsistema de saúde indígena, } \\
\text { evidenciando a sua evolução } \\
\text { no processo histórico e legal. }\end{array}$ & $\begin{array}{l}\text { Ficou evidente as recorrentes } \\
\text { disparidades ao analisarmos a saúde } \\
\text { bucal indígena em comparação à } \\
\text { população brasileira não indígena. Ao } \\
\text { longo do tempo, marcadamente pela } \\
\text { descontinuidade das políticas públicas } \\
\text { que impediram a integralidade das ações } \\
\text { de saúde bucal. }\end{array}$ \\
\hline $\begin{array}{l}\text { Epidemiological profile } \\
\text { of caries and need for } \\
\text { dental extraction in a } \\
\text { Kaingang adult } \\
\text { Indigenous population } \\
\text { (Soares et al.) }\end{array}$ & 2019 & $\begin{array}{c}\text { Estudo Transversal } \\
\text { Descritivo }\end{array}$ & $\begin{array}{l}\text { Avaliar a prevalência e a } \\
\text { gravidade da cárie dentária, } \\
\text { além dos fatores associados à } \\
\text { necessidade de extração } \\
\text { dentária entre os indígenas } \\
\text { Kaingang adultos. }\end{array}$ & $\begin{array}{l}\text { Com o total de } 107 \text { indígenas adultos } \\
\text { examinados. A região anterior inferior } \\
\text { apresentou as maiores taxas de dentes } \\
\text { hígidos, enquanto os primeiros molares } \\
\text { inferiores apresentaram os } \\
\text { mais baixo, a necessidade de extração } \\
\text { dentária foi observada em } 34,58 \% \text {. Os } \\
\text { dados colhidos indicam que as altas } \\
\text { frequências de cárie são reflexo da } \\
\text { assistência inadequada. }\end{array}$ \\
\hline
\end{tabular}

Fonte: Autores (2020).

\section{Discussão}

Segundo Bertanha et al. (2012), os primeiros registros de assistência à saúde para comunidades indígenas foram realizados através de missões religiosas, marcadamente pelo contato truculento. O atendimento odontológico por sua vez, passou a ser registrado apenas, em 1956, com as ações sanitárias do médico e indigenista Noel Nutes, embora são escassos os registros.

Em consonância ao exposto, Rodrigues et al. (2018), afirmam que o panorama histórico de implementação das estratégias de políticas públicas voltadas a população indígena, está diretamente associado a dissidências e descontinuidades. 
Somente a partir da implementação da Programa Federal Brasil Sorridente, em 2004, que obteve êxitos consideráveis para a saúde bucal brasileira, entretanto as estratégias em favor dos indígenas eram práticas pontuais e localizadas.

Este cenário de negligência com a oferta de programas de saúde direcionados a esses povos, reverberam também nos resultados ruins da auto avaliação de saúde bucal, como apontam Arantes e Frazão (2018), sobre os relatos de dor de dente, dificuldade para mastigar e alterações na fala devido a problemas dentários, que foram os resultados mais prevalentes em seu estudo.

Rigonatto, Antunes e Frazão (2001); Arantes et al. (2009) \& Lemos et al. (2018), asseveram que os levantamentos epidemiológicos em saúde bucal permitem o conhecimento de informações relevantes sobre o estado de saúde de uma determinada população, fornecendo os subsídios para o planejamento de ações mais assertivas e facilitando a monitoração dos indicadores. Entretanto, a irregularidade observada nos serviços programados para estas comunidades, têm exercido a indesejável consequência de ampliar as desigualdades.

Em concordância com estes estudos, Fratucci (2000); Arantes, Santos e Coimbra (2001) \& Sampaio et al. (2010), apontam que as amostras investigadas caracterizadas pela elevada presença de cárie, perda dentária, periodontopatias e extensa necessidade de tratamento odontológico, demonstram que a ausência da implantação de propostas de atenção à saúde bucal, seja a responsável pela evolução exponencial desses índices. Somado a isso, Maurício (2012); Filho, Santos e Vettore (2013), reforçam que o desenvolvimento de políticas públicas de saúde específicas precisa considerar as particularidades socioculturais desses povos, não podendo ser analisadas de forma generalizadas e homogêneas.

Ao avaliar a correlação da experiência de cárie entre mães e filhos em uma comunidade indígena, Hirooka et al. (2014), relataram alta prevalência de cárie dentária em crianças e consideráveis perdas dentárias nas mães, além disso, a experiência materno-infantil de cárie dentária tendeu a aumentar com a idade. O que sugere a importância do envolvimento de toda comunidade no planejamento das estratégias de prevenção da cárie, e não apenas a mãe, no que se refere à saúde bucal de crianças indígenas.

Carneiro (2005), aponta que o outro fator decisivo para a precarização da condição bucal das comunidades indígenas brasileira, é a mudança nos padrões da dieta e aporte nutricional. Moura, Batista e Moreira (2010), atestam que o contato com a população ocidentalizada, juntamente com seus hábitos alimentares, introduzindo o consumo de produtos industrializados, em particular o açúcar refinado, bem como as mudanças no preparo dos alimentos que passaram a ser consumidos cozidos, de consistência mole, facilitando a adesão do biofilme bacteriano de alto potencial cariogênico aos elementos dentários.

Caires et al. (2018) apontaram os fatores supracitados como determinantes para o aumento da experiência de cárie, que invariavelmente corrobora em maiores indicações de tratamento endodôntico. Esta situação é responsável não só por evidenciar os quadros de dor de dente, experiência marcadamente das classes sociais menos favorecidas, como também associada aos resultados alarmantes de dentes com indicação de exodontia ou já extraídos em função da cárie e da falta de condições para a realização de um tratamento conservador (Arantes, Santos e Frazão, 2010; Soares et al., 2019).

Com relação a fluorose dentária e condição periodontal, Alencar et al. (2011), avaliou 98 crianças e adolescentes aldeadas de 05 a 12 anos, observou-se que a presença de sangramento gengival esteve ausente em mais da metade da população aos 5 anos de idade, contudo foi detectado que aos 12 anos a prevalência de alterações gengivais era de $68,3 \%$. Com relação à fluorose dentária, a prevalência é pequena, estando presente nos graus mais leves, embora não haja outros estudos para que os dados possam ser confrontados.

No que se refere às forças mastigatórias, Regalo et al. (2008), acarearam a força de mordida de indivíduos indígenas com um grupo não indígena, onde os resultados revelaram uma propensão da força de mordida máxima ser maior no grupo indígena. Entretanto, os estudos acerca das oclusopatias, apontem uma baixa prevalência de má oclusão entre as comunidades indígenas, Arantes, Santos e Coimbra (2001) \& Campos et al. (2017), advogam que a falta de padronização metodológica e o 
número reduzido de estudos específicos, são responsáveis pela ausência de uma avaliação minuciosa sobre oclusopatias em povos indígenas.

No tocante da estomatopatologia, apenas um trabalho dedicou-se a este levantamento, ao analisar 291 índios, de oito etnias Mato-Grossenses, de ambos os sexos, com idades variando de 1 a 96 anos, sendo identificadas 132 variações da normalidade, onde a anquiloglossia esteve presente em 37,1\% destas variações, seguida de glossite migratória benigna em $1,7 \%$ dos casos, tórus mandibular e candidíase coresponderam a $1 \%$ das alterações observadas, nenhuma lesão maligna foi identificada nestes grupos relatados por Vieira et al. (2011).

Por fim, Bulgarelli et al. (2012), afimam que quando busca-se identificar modelos eficientes de saúde num contexto sociocultural indígena, para além dos números precários de profissionais que atuam com esses grupos, há uma necessidade urgente de que tais profissionais estejam capacitados para ofertar um serviço acertadamente responsável, menos etnocêntrico e capaz de aproveitar os conhecimentos locais previamente empregados pela comunidade. Deste modo, é imperativo que as experiências extramuros estejam presentes desde a formação universitária dos futuros profissionais de saúde.

Desta forma, os trabalhos analisados revelaram uma tendência de deterioração exponencial das condições de saúde bucal dos grupos indígenas brasileiros, que estão diretamente associadas a aspectos contextuais e coletivos, embora permaneçam largamente desconhecidos, o que precisa ser revertido. Por fim, é latente a necessidade do desenvolvimento de programas mais estruturados e fixos especificamente para as comunidades indígenas, visto que as desigualdades encontradas são reflexos da ineficiência do acesso a utilização de serviços de atenção à saúde.

\section{Considerações Finais}

Em virtude dos resultados analisados, podemos concluir que o perfil epidemiológico da saúde bucal dos povos indígenas brasileiros nos últimos vinte anos, apresenta-se precário e complexo. Embora restem poucas dúvidas de que as condições de saúde desses povos são débeis, o que os colocam em desvantagem em relação a outros grupos da sociedade nacional. A escassez de estudos mais robustos, atrelados a falta de padronização metodológica aplicada nos trabalhos atuais, inviabiliza substanciar tais questões de maneira minuciosa e precisa.

Dado o exposto, é imprescindível que mais levantamentos epidemiológicos voltados a população indígena sejam realizados, a fim de caracterizar as reais condições e necessidades desses povos, bem como acompanhá-las ao longo do tempo. Por fim, é urgente a implementação de programas preventivos e aumento da cobertura do atendimento odontológico, pautados nas especificidades étnico-culturais e seus aspectos gerais de saúde.

\section{Referências}

Alencar, C. O., Miclos, P. V., Carvalho, R. B., \& Cortelli, J. R. (2011). Fluorose dentária e condição periodontal de crianças indígenas do município de Aracruz, ES. Braz J Periodontol, 21(4), 80-85.

Arantes, R., Santos, R. V., \& Coimbra, C. E. A. (2001). Saúde bucal na população indígena Xavánte de Pimentel Barbosa, Mato Grosso, Brasil. Cad. Saúde Pública, 7(2), 375-384.

Arantes, R., Santos, R. V., Frazão, P., \& Coimbra, C. E. A. (2009). Caries, gender and socio-economic change in the Xavante Indians from Central Brazil. Annals of Human Biology, 36(2), 162-175.

Arantes, R., Santos, R. V., \& Frazão, P. (2010). Oral health in transition: the case of Indigenous peoples from Brazil. International Dental Journal, 60(3), 235240. 
Arantes, R., \& Frazão, P. (2018). Subjective oral symtoms associated with self-rated oral heath among indigenous groups in Central-West Brazil. Communit Dent oral Epidemiol, 00, 1-8.

Baldisserotto, J., Ferreira, A. M., \& Warmling, C. M. (2019). Condições de saúde bucal da população indígena guarani moradora no Sul do Brasil. Cad. Saúde Colet, 27(4), 468-475.

Bertanha, W. F. F., Cavalcante, G. M. S., Cavalcante, A. L., Arruda, T. A., \& D’Ávila, S. (2012). Atenção à saúde bucal nas comunidades indígenas: evolução e desafios - uma revisão de literatura. $R$ bras ci Saúde, 16(1), 105-112.

Bulgarelli, A. F., Roperto, R. C., Mestriner, S. F., \& Junior, W. M. (2012). Destitry students perceptions about na extramural experience with a Brazilian indigenous community. Indian J Dent Res, 23, 498-500.

Caires, N. C. M., Brito, L. C. N., Vieira, L. Q., \& Sobrinho, A. P. R. (2018). Epidemiological analysis and need for endodontic treatment among the indigenous Santaré-Mawé and Tikuna. Braz Oral Res, 32(19), 1-8.

Campos, J. N. S., Santos, D. C. L., Negrete, D., Flaiban, E., \& Lemos, P. N. (2016). Oclusão dentária em populações indígenas. Rev Odontol Univ Cid São Paulo, 29(1), 57-65.

Carneiro, M. C. G., Santos, R. V., Garnelo, L., Rebelo, M. A. B., \& Coimbra, C. E. A. (2008). Cárie dentária e necessidade de tratamento odontológico entre os índios Baniwa do Alto Rio Negro, Amazonas. Ciência \& Saúde Coletiva, 13(6), 1895-1992, 2008.

Ferreira, D. C., Gonçalves, T. R., Celeste, R. K., Olinto, M. T. A., \& Pattussi, M. P. (2020). Aspectos psicossociais e percepção de impacto da saúde bucal na qualidade de vida em adultos do Sul do Brasil. Rev Bras Epidemiol, 23, 1-13.

Filho, P. A., Santos, R. V., \& Vettore, M. V. (2013). Desigualdades socioambientais na ocorrência de cárie dentária na população indígena no Brasil: evidências entre 2000 e 2007. Rev Bras Epidemiol, 16(3), 692-704.

França, M. A. S. A., Freire, M. C. M., Pereira, E. M., \& Marcelo, V. C. (2020). Indicadores de saúde bucal propostos pelo Ministério da Saúde para monitoramento e avaliação das ações no Sistema Único de Saúde: pesquisa documental, 2000-2017. Epidemiol. Serv. Saude, Brasília, 29(1), 1-9.

Fratucci, M. V. B. (2000). Alguns aspectos das condições de saúde bucal de uma população indígena Guarani Mbyá no município de São Paulo. Dissertação (Mestrado em Saúde Pública) - Faculdade de Saúde Pública, Universidade de São Paulo. São Paulo.

Galea, S., \& Vaughan, R.D. (2019). Public Health and Marginalized Populations: A Public Health of Consequence. Am J Public Health, 109(10), 1327-1328.

Garnelo, L., Brandão, L. C., \& Levino, A. (2005). Dimensões e potencialidades dos sistemas de informação geográfica na saúde indígena. Rev Saúde Pública, 39(4), 634-640.

Hirooka, L. B., Junior, W. M., Mestriner, S. F., Nunes, S. A. C., Lemos, P. N., \& Franco, L, J. (2014). Dental caries in mother-child pairs from Xingu. Braz J Oral Sci, 13(1), 43-46.

Junior, J. R. L. S., Silva, E. D. A., Silva, E. C. B., Lemos, J. F. S., Junior, R. M. V., Silva, J. M. D. S., Nemezio, M. A., Junior, V. E. S., Porto, I. C. C. M., \& Romão, D. A. Odontologia aplicada à saúde da população indígena do Brasil: uma revisão integrativa. Research, Society and Development, 9(11), 1-13.

Lemos, P. N., Rodrigues, D. A., Frazão, P., Hirooka, L. B., Guisilini, A. C., \& Narvai, P. C. (2018). Atenção à saúde bucal no parque indígena do Xingu, Brasil, no período de 2004-2013: um olhar a partir de indicadores de avaliação. Cad Saúde Pública, 34(4).

Lima, K. E. R., Gondim, G. F., Santana, G. S., Silva, F. G. O., \& Silva, C. H. F. (2020) Attention to oral health for indigenous peoples in brazil: an integrative review. Braz. J. of Develop, 6(4), 18704-18713. 
Maurício, H. A. (2012). A saúde bucal do povo indígena Xukuru do Ororubá da faixa etária de 10 a 14 anos. Dissertação (Mestrado acadêmico em saúde pública). Recife: Fundação Oswaldo Cruz.

Maurício, H. A., \& Moreira, R. D. (2014). Condições de saúde bucal da etnia Xukuru do Ororubá em Pernambuco: análise multinível. Rev Bras Epidemiol, $787-800$.

Moura, P. G., Batista, L. R. V., \& Moreira, E. A. M. (2010). População indígena: uma reflexão sobre a influência da civilização urbana no estado nutricional e na saúde bucal. Rev. Nutr, 23(3), 459-465.

Nicacio, K., Cavalcante, G. C., Martins, K., Barbosa, R., Gonçalves, S., Lins, M. A., \& Melo, M. (2019). Saúde pública voltada para a população indígena. Ciências Humanas e Sociais, 5(3), 75-84.

Netto, M. F. V., \& Chicon, J. B. (2020). Expedição Amazônia - Promoção de saúde bucal com a população ribeirinha. Revista Científica FAESA, 16(1), 2531.

Neves, M., Giordani, J. M. A., \& Hugo, F. N. (2019). Primary dental healthcare in Brazil: the work process of oral health teams. Ciência \& Saúde Coletiva, 24(5), 1809-1820.

Regalo, S. C. H., Santos, C. M., Vitti, M., Regalo, C. A., Vasconcelos, P. B., Mestriner, W., Semprini, M., Dias, F. J., Hallak, J. E. C., \& Siéssere, S. (2008). Evaluation of molar and incisor bite force in indigenous compared with white population in Brazil. Archives of oral biology, 53(1), $282-286$.

Rigonatto, D. D. L., Antunes, J. L. F., \& Frazão, P. (2001). Dental caries experience in indians of the upper Xingu, Brazil. Rev. Inst. Med. Trop, 43(2), 93-98.

Rodrigues, F. I., Garbin, C. A. S., Moimaz, S. A. S., \& Saliba, N. A. (2018). Análise documental dos serviços de saúde bucal ofertados à população indígena no Brasil. Revista Ciência Plural, 4(1), 7-21.

Sampaio, F. C., Freitas, C. H. S. M., Cabral, M. B. F. \& Machado, A. T. A. B. (2010). Dental caries and treatment needs among indigenous people of the Potiguara Indian reservation in Brazil. Rev Panam Salud Publica, 27(4), 246-251.

Silva, F. M. (2019). Os discursos étnico raciais no processo de construção da identidade indígena na Amazônia. Muiraquitã, 7(1).

Soares, G. H., Aragão, A. S., Frias, A. C., Werneck, R. I., Biazevic, M. G. H., \& Crosato, E. M. (2019). Epidemiological profile of caries and need for dental extraction in a Kaingang adult indegenous population. Rev Bras Epidemiol, 22(1), 1-13.

Souza, M. T., Silva, M. D., \& Carvalho, R. (2010). Revisão integrativa: o que é e como fazer. Rev Einstein, 8(1), $102-6$.

Souza, M. F., Prado, E. A. J., Leles, F. A. G., Andrade, N. F., MArzola, R. F., Barros, F. P. C., \& Mendonça, A. V. M. (2019). Potencialidades da Atenção Básica à Saúde na consolidação dos sistemas universais. Saúde Debate, 43(5), 82-93.

Tavares, S. S., Melo, A. S., Stefani, C. M., \& Jr. Pucca, G. A. (2020). O Brasil Sorridente aos olhos da $3^{\text {a }}$ Conferência Nacional de Saúde Bucal e da $16^{\circ}$ Conferência Nacional de Saúde. Tempus Actas de Saúde Colet, 14(1), 127-142.

Vieira, E. M. M., Ciesielski, F. I. N., Gaetti-Jardim, E. C., Hespanhol, D., Castro, E. V. F. L., Castro, A. L., Schweitzer, C. M., \& Junior, E. G. J. (2011). Evaluation of oral health in a community of native Brazilians of the Umutina reservation, Mato Grosso state. Int. J. Odontostomat, 5(1), 59-63.

Kabad, J. F., Pontes, A. L. M., \& Monteiro, S. (2020). Relações entre produção cientifica e políticas públicas: o caso da área da saúde dos povos indígenas no campo da saúde coletiva. Ciência \& Saúde Coletiva, 25(5), 1653-1665.

Wenczenovicz, T. J. (2018). Saúde Indígena: Reflexões Contemporâneas. Cad. Ibero-Amer. Dir. Sanit, 7(1), 63-82. 